\title{
Calculadora para dimensionar sistemas fotovoltaicos interconectados
}

\section{Calculator for dimensioning interconnected photovoltaic systems}

FLORES-RAMIREZ, Oscar†*, JIMENEZ-AGUAS, Jimmy, HUESCA-LAZCANO, Erick Eduardo y ROMERO-RODRIGUEZ, Gabriel

Universidad Politécnica de Amozoc, Departamento de Ingeniería en Energía, México.

ID $1^{\mathrm{er}}$ Autor: Oscar, Flores-Ramírez / ORC ID: 0000-0001-9884-9499, Research ID Thomson: E-8242-2018, Arxiv ID Author: 429567-444L3Z-UIWFRF, CVU CONACYT ID: 92914

ID $1^{\mathrm{er}}$ Coautor: Jimmy, Jimenez-Aguas / ORC ID: 0000-0001-9345-464X

ID $2^{\text {do }}$ Coautor: Erick Eduardo, Huesca-Lazcano / ORC ID: 0000-0002-0505-8442, Research ID Thomson: F-1162-2018, Open ID: 357590366000, CVU CONACYT ID: 223342

ID $3^{\text {er }}$ Coautor: Gabriel, Romero-Rodríguez / ORC ID: 0000-0002-5124-3016, Research ID Thomson: Y-2756-2018, CVU CONACYT ID: 299737

DOI: $10.35429 / J U S D .2020 .19 .6 .31 .41$

Recibido: 25 de Julio, 2020; Aceptado 30 de Diciembre, 2020

\section{Resumen}

La generación de energía eléctrica por medio de la energía solar fotovoltaica se ha convertido en lo más rentable en la actualidad. Lo más importante de un ingeniero en energía son los cálculos, por lo cual se ha diseñado una calculadora automatizada en Excel, donde se resumen los cálculos de un dimensionamiento para un sistema fotovoltaico interconectado a la red de energía eléctrica, incluye los análisis de facturación de la tarifa en que se encuentre y en TARIFA DAC (Domestico de Alto Consumo), TARIFA 01, con el objetivo de poder ahorrar tiempo, y dinero, cuando se realice el cálculo y el estudio económico, es de alta importancia conocer este tipo de información ya que las personas solo venden proyectos sin conocimiento alguno y dañan la reputación del área profesional, con esta calculadora automatizada de Excel cualquier persona con conocimientos básicos podrá manipularla sin problema alguno, dando un servicio de excelencia ahorrando demasiado tiempo en correcciones donde eso cuesta dinero, el propio sistema explica de manera gráfica la generación del SFVI, estudio económico y tiempo de recuperación, el cliente tendrá un amplio conocimiento por lo que paga.

Energía solar, Sistemas fotovoltaicos, Calculadora automatizada, Tarifas

\begin{abstract}
The generation of electrical energy by means of photovoltaic solar energy has become the most profitable today. The most important thing for an energy engineer are the calculations, for which an automated calculator in Excel has been designed, where the calculations of a dimensioning for a photovoltaic system interconnected to the electrical power network are summarized, including billing analyzes of the rate you are in and the DAC RATE (High Consumption Domestic), RATE 01, in order to save time and money, when calculating and studying economically, it is highly important to know this type of information Since people only sell projects without any knowledge and damage the reputation of the professional area, with this automated Excel calculator anyone with basic knowledge can manipulate it without any problem, giving an excellent service saving too much time on corrections where that costs money, the system itself graphically explains the generation of the SFVI, economic study and recovery time, the client will have a broad knowledge of what you pay for.
\end{abstract}

Solar energy, Photovoltaic systems, Automated calculator, Rates

Citación: FLORES-RAMIREZ, Oscar, JIMENEZ-AGUAS, Jimmy, HUESCA-LAZCANO, Erick Eduardo y ROMERORODRIGUEZ, Gabriel. Calculadora para dimensionar sistemas fotovoltaicos interconectados. Revista del Desarrollo Urbano y Sustentable. 2020. 6-19:31-41.

\footnotetext{
*Correspondencia al autor (Correo electrónico: oscar.flores@upamozoc.edu.mx)

$\dagger$ Investigador contribuyendo como primer autor.
} 


\section{Introducción}

La energía eléctrica generada por medio de la tecnología solar es lo más eficiente en la actualidad por lo que se ha considerado unas de las principales fuentes de energía más importante. México es uno de los países con más alto índice de radiación solar donde solo se aprovecha el $5 \%$.

La cual es distribuida en diferentes aplicaciones y usos como por ejemplo en la generación de energía eléctrica, calentamiento de agua, calefacción y refrigeración, etc., que son algunas de las necesidades básicas del ser humano.

Así se ayuda a la naturaleza a que se restablezca de manera más rápida que de lo estimado, es donde cambiamos un árbol por un calentador solar, donde reducimos plantas nucleares por paneles fotovoltaicos, el tema de los paneles solares es muy polémico por lo que es una tecnología muy prometedora lo cual hace énfasis en un ahorro económico una vez instalado el sistema ya sea autónomo o interconectado a red, dependerá del proyectista o de la persona encargada del proyecto fotovoltaico; ya que será quien realice los cálculos de manera exacta, es por eso que se diseñó una calculadora automatizada en Excel para que se puedan apoyar los proyectistas que estén comenzado adentrarse al mercado y no solo para ellos sino también para aquellos que llevan tiempo; por lo cual se añaden algunas comparaciones en facturaciones del cliente durante un cierto periodo si continua con su hábito energético, lo que siempre hay que hacer es "tomar la decisión correcta en el momento correcto" y esa es la función principal del proyectista, no solo eso sino que también nos dirá en que tiempo se recupera la inversión del proyecto de una forma más practica por lo que tiene un área de gráficos donde se hace más digerible, en la presentación del proyecto energético es donde se puede calcular desde la TARIFA01 a la TARIFA DAC por lo que son tarifas con más índice de uso y a la vez preocupantes donde con poco consumo pagas demasiado.

\section{Planteamiento del problema}

Uno de los parámetros para medir la calidad de los proyectos es realizar un buen cálculo, pero en ocasiones no se cuenta con el tiempo adecuado ni con la experiencia y se corre el riesgo que no compren dicho proyecto y que solo sea un tiempo mal invertido o simplemente no se cuente con la habilidad verbal para comunicarle al cliente los beneficios que contara con nuestro servicio, por lo cual el programa está diseñado con gráficos para que sea más práctico y quien compre el proyecto solar tenga la seguridad de solventar sus necesidades eléctricas, el mercado laboral del área solar ha crecido de manera exponencial debido que se conoce de manera errónea que es práctico pero la realidad no es así, por lo que en un buen proyecto se tiene un proceso administrativo, técnico e ingenieril donde no todos conocen, por lo que muchos lo hacen de manera empírica, poniendo en riesgo al personal de la empresa o hasta la empresa propia por no llevar una proceso establecido, el riesgo de un proyecto que se convierta en una cotización es muy alto y solo eso nos trae perdidas de todo tipo, con la calculadora automatizada solo se anexarían las variables que pide el sistema y de manera rápida tendríamos un preestablecido precio fijo.

\section{Justificación}

Un hecho real es el cambio climático, que se está generando por el consumo energético de los combustibles fósiles (petróleo, carbón y gas), sin darnos cuenta de que este consumo energético es insostenible, debido a que los yacimientos de combustibles se están agotando; adicionalmente está ocasionando graves trastornos ambientales deteriorando el planeta. para nadie es un secreto que el calentamiento global es un problema actual y a nivel mundial algunas naciones empiezan a cambiar sus formas de producción y sus normas con el fin de tratar de disminuir los efectos de su industria en el ambiente, así mismo se buscan formas de producción de energía alternativas, pues es ya sabido que el gran problema gira entorno a la liberación de bióxido de carbono $\left(\mathrm{CO}_{2}\right)$ producido por la quema de esos combustibles que son generados por los medios de transporte, los electrodomésticos y las industrias. 


\section{Objetivo general}

- Dar a conocer las diferencias que existen entre la tarifa DAC y 01, con el programa Excel para determinar la viabilidad del proyecto.

\section{Objetivos específicos}

- Dimencionar el sistema fotovoltaico.

- Realizar el estudio económico.

- Comparar los resultados obtenidos de manera grafica.

- Determinar la viabilidad del proyecto a ejecutar.

- Dar a conocer los resultados obtenidos.

\section{Marco Teórico}

Como resultado de la Reforma Energética, la Comisión Reguladora de Energía creó un nuevo esquema tarifario que entró en vigor a partir del 1 de diciembre de 2017; excluyendo las tarifas domésticas, agrícolas y acuícolas que continúan con el esquema anterior.

Las composiciones de las nuevas tarifas incluyen los cargos fijos del suministrador y cargos variables que dependen de condiciones de capacidad y de generación, dependiendo del tipo de servicio que se necesita, ya sea para negocio o industria.

\begin{tabular}{|l|l|l|}
\hline \multicolumn{1}{|c}{ Tipo } & \multicolumn{1}{c|}{$\begin{array}{c}\text { Nueva } \\
\text { categoria }\end{array}$} \\
\hline $\begin{array}{l}\text { General baja } \\
\text { tensión }\end{array}$ & Pdbt & $\begin{array}{l}\text { Pequeña demanda } \\
\text { (hasta 25kw-mes). }\end{array}$ \\
\hline $\begin{array}{l}\text { General baja } \\
\text { tensión }\end{array}$ & Gdbt & $\begin{array}{l}\text { Gran demanda } \\
\text { (mayor 25kw-mes } \\
\text { en media tensión). }\end{array}$ \\
\hline $\begin{array}{l}\text { General media } \\
\text { tensión }\end{array}$ & Gdbto & $\begin{array}{l}\text { Gran demanda } \\
\text { (mayor 25kw-mes } \\
\text { en media tensión } \\
\text { ordianria). }\end{array}$ \\
\hline $\begin{array}{l}\text { General media } \\
\text { tensión }\end{array}$ & Gdbth & $\begin{array}{l}\text { Gran demanda } \\
\text { (mayor 25kw-mes } \\
\text { en media tensión } \\
\text { horaria. }\end{array}$ \\
\hline $\begin{array}{l}\text { General media } \\
\text { tensión }\end{array}$ & Aptm & $\begin{array}{l}\text { Alumbrado } \\
\text { público en media } \\
\text { tensión. }\end{array}$ \\
\hline $\begin{array}{l}\text { General media } \\
\text { tensión }\end{array}$ & Ramt & $\begin{array}{l}\text { Riego agricola en } \\
\text { media tensión. }\end{array}$ \\
\hline $\begin{array}{l}\text { General alta } \\
\text { tensión }\end{array}$ & Dist & $\begin{array}{l}\text { Demanda } \\
\text { industrial en } \\
\text { Subtransmisión. }\end{array}$ \\
\hline
\end{tabular}

\begin{tabular}{|l|l|l|}
\hline $\begin{array}{l}\text { General alta } \\
\text { tensión }\end{array}$ & Dit & $\begin{array}{l}\text { Demanda } \\
\text { industrial en } \\
\text { subtransmisión. }\end{array}$ \\
\hline $\begin{array}{l}\text { Especifica } \\
\text { baja tensión }\end{array}$ & Rabt & $\begin{array}{l}\text { Riego agricola en } \\
\text { baja tensión. }\end{array}$ \\
\hline $\begin{array}{l}\text { Especifica } \\
\text { baja tensión }\end{array}$ & Apbt & $\begin{array}{l}\text { Riego agricola en } \\
\text { baja tensión. }\end{array}$ \\
\hline
\end{tabular}

Tabla 1 Clasificación de tarifas Fuente: CFE.com.mx

\section{¿Qué es la tarifa Doméstica de Alto Consumo (DAC)?}

Es la tarifa que se aplica a los servicios domésticos que registran mayor consumo mensual del límite superior promedio permitido por las tarifas domésticas que se aplican en tu localidad.

¿Cómo se determina el consumo mensual superior promedio?

Se determina con el promedio del consumo mensual del servicio doméstico en un año móvil (últimos 365 días facturados).

Límite superior promedio de consumo mensual antes de ser reclasificado a tarifa DAC, por localidad

Tarifa 1: 250 (doscientos cincuenta) kWh/mes.

Tarifa 1A: 300 (trescientos) kWh/mes.

Tarifa 1B: 400 (cuatrocientos) kWh/mes.

Tarifa1C:850(ochocientos cincuenta) kWh/mes.

Tarifa 1D: 1,000 (un mil) kWh/mes.

Tarifa 1E: 2,000 (dos mil) kWh/mes.

Tarifa 1F: 2,500 (dos mil quinientos) $\mathrm{kWh} / \mathrm{mes}$

"Es importante saber que ésta tarifa no cuenta con el apoyo gubernamental (conocido como subsidio), por lo que, si se llega a reclasificar la tarifa doméstica a DAC, se incrementaría el cobro de la factura." 


\section{¿Qué es la Tarifa 01?}

La tarifa 01 se aplicará a todos los servicios que destinen la energía para uso exclusivamente doméstico, para cargas que no sean consideradas de alto consumo de acuerdo a lo establecido en la Tarifa DAC, conectadas individualmente a cada residencia, apartamento, apartamento en condominio o vivienda.

\section{Concepto de Excel}

Es un programa informático desarrollado que permite realizartareas contables y financieras gracias a sus funciones, desarrolladas específicamente para ayudar a crear y trabajar con hojas de cálculo.

\section{¿Qué es la energía solar fotovoltaica?}

La energía solar fotovoltaica transforma de manera directa la luz solar en electricidad empleando una tecnología basada en el efecto fotovoltaico. Al incidir la radiación del sol sobre una de las caras de una célula fotoeléctrica (que conforman los paneles) se produce una diferencia de potencial eléctrico entre ambas caras que hace que los electrones salten de un lugar a otro, generando así corriente eléctrica, como se muestra en la figura 1.

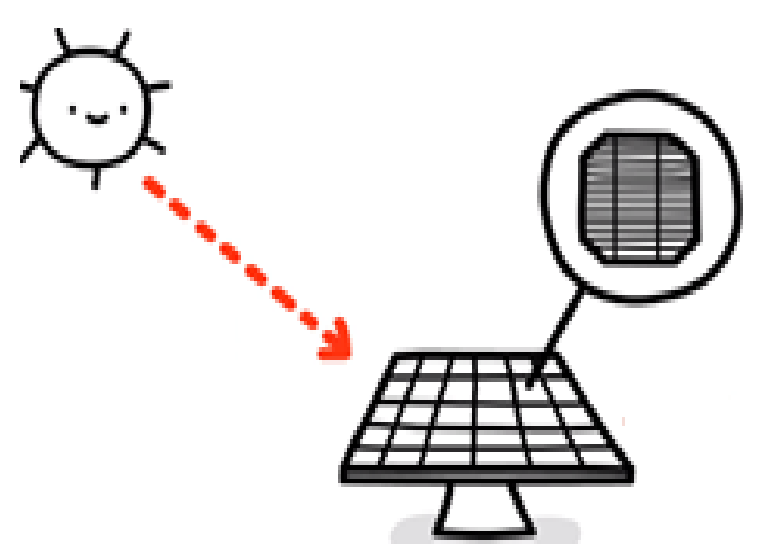

Figura 1 Proceso de generación de electricidad Fuente: acciona.com

\section{¿Beneficios de la energía fotovoltaica?}

La energía eléctrica generada mediante paneles solares fotovoltaicos es inagotable $y$ no contamina, por lo que contribuye al desarrollo sostenible, además de favorecer el desarrollo del empleo local.
Punto de equilibrio, es uno de los elementos centrales en cualquier tipo de negocio pues nos permite determinar el punto de equilibrio y es uno de los elementos centrales en cualquier tipo de negocio pues nos permite determinar el nivel de ventas necesario para cubrir los costes totales $\mathrm{o}$, en otras palabras, el nivel de ingresos que cubre los costes fijos y los costes variables, el nivel de ventas necesario para cubrir los costes totales o, en otras palabras, el nivel de ingresos que cubre los costes fijos y los costes variables en este caso las variables serán el tiempo y costo del proyecto, como se muestra en la figura 2.

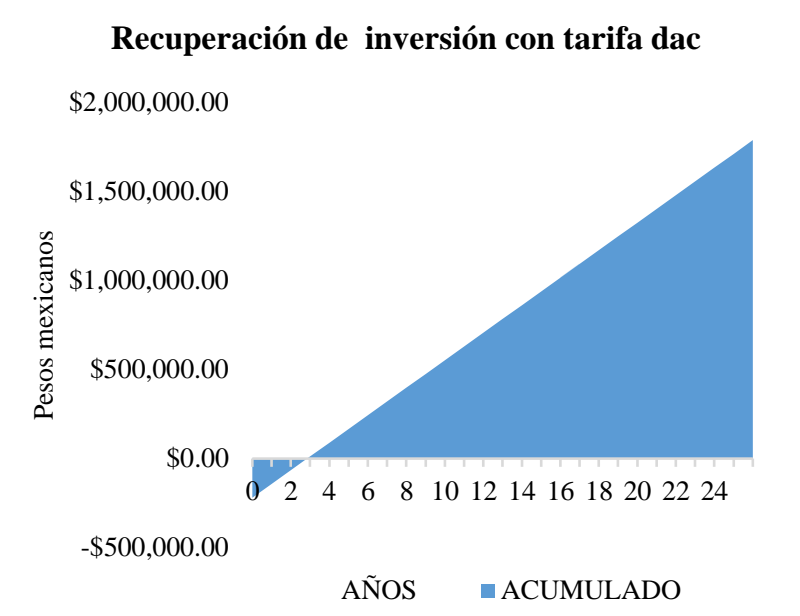

Figura 2 Punto de equilibrio de un proyecto Fuente: Elaboración propia

\section{Datos de facturación}

Es el apartado del recibo de luz donde incluye:

Concepto: es el resultado de aplicar las cuotas que específicamente señala cada tarifa al consumo en sus diferentes rangos:

Básico: depende de la localidad, los primeros kilowatts hora que consume, 150 generalmente, tendrán un precio más bajo eso es decir que tendremos mayor apoyo del subsidio gubernamental.

Intermedio: si ya has rebasado el consumo básico, los kilowatts hora que siguen se cobran a un precio mayor y recibirás una cantidad de subsidio menor. 
Excedente: si ya has rebasado el consumo intermedio aparecerá otro cargo bajo el nombre de excedente. El precio de estos kilowatts hora extra será bastante más alto. Si tu consumo pasa el promedio de Kwh de tu región en cada periodo durante los últimos seis bimestres se aplicará una tarifa de alto consumo en la que ya no se contemplan subsidios.

Lectura actual: Indica la cantidad de $\mathrm{kWh}$ registrado en tu medidor; es la lectura más reciente.

Lectura anterior: Indica la cantidad de $\mathrm{kWh}$ registrado en tu medidor al final del bimestre anterior.

Total del periodo: Es la diferencia entre la lectura anterior y la actual e indica el número de kWh que consumió el último bimestre.

Precio: Es el precio por $\mathrm{kWh}$ en cada uno de los rangos de las tarifas.

Subtotal: Es el precio que sale de multiplicar el costo de la energía por el consumo en cada uno de los rangos.

Nuevo Odometro: El cliente visualiza el comportamiento de sus consumos a fin de evitar caer en tarifa DAC.

\section{Desglose del importe a pagar}

Energía: Es el resultado de aplicar las cuotas que específicamente señala cada tarifa, al consumo de sus diferentes conceptos, punta, semipunta y base.

IVA: Es la tasa en proporción que se traslada a los usuarios, autorizada por la secretaria de hacienda y crédito público y que aplica a la facturación de energía.

\section{DAP: Derecho de Alumbrado Público.}

Cargos o créditos: son los diversos conceptos de pago que se pueden incluir en el aviso del recibo.

Facturación del periodo: la suma de la energía más el IVA (16\%)

Adeudo Anterior: Total facturado del periodo anterior.
Pago anterior: pago realizado en el periodo anterior.

Total a pagar: Total del adeudo al periodo actual.

En la tabla 2, se muestran las tarifas vigentes.

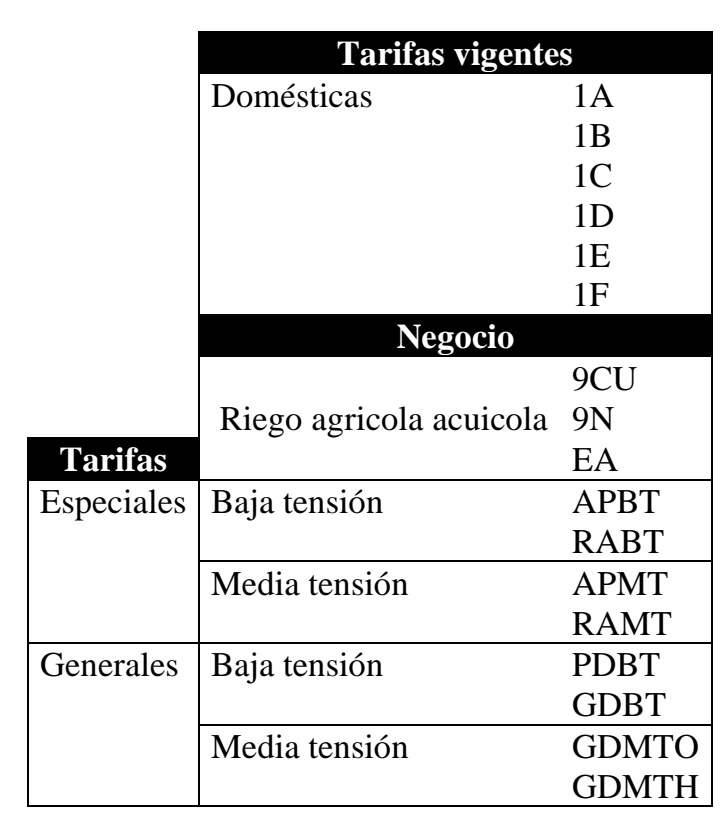

Tabla 2 Tarifas más usadas Fuente: Elaboración propia

\section{Metodología}

\section{Descripción del método}

Para obtener los estudios económicos con sus futuros pagos del proyecto se requiere seguir un simple proceso de optimización para una obtención de resultados.

Para la realización de este proyecto el método consistió en los siguientes pasos:

- Obtener el historial de pagos del cliente.

- Descargar los recibos emitidos ante CFE de un año atrás.

- Revisar qué tipo de facturación es, puede que sean mensuales o bimestrales.

- Hallar la ubicación por medio de Google maps.

- Obtener la radiación que incide en el lugar. En caso de no poder obtener ese dato lo que se puede hacer es tomar el dato del estado y no del municipio. 
- Poner las fechas correspondientes de manera bimestral como mensual.

- Encontrar los siguientes datos en el recibo, Bimestre, consumo en $\mathrm{kWh}$, cargos fijos (solo para DAC) DAP (puede aplicar para tarifa DAC y 01). Básico (solo para DAC), y energía (tarifa 01).

- Poner los siguientes datos del recibo de CFE a la tabla de Excel, Bimestre = Bimestre consumo en $\mathrm{kWh}=$ consumo en $\mathrm{kWh}$.

Cargos fijos $=$ Cargos fijos

$\mathrm{DAP}=\mathrm{DAP}$

Básico= facturación sin IVA con CFE

Energía $=$ facturación sin IVA con CFE

Mes $=$ Mes

Días del mes $=$ días

Radiación $=$ Radiación

- Modificar los datos.

\section{- Formula:}

Días del mes $=$ Dm.

$\mathrm{HSP}=$ horas solares pico.

Wpanel= Potencia del panel (w).

$\mathrm{Np}=$ Numeros de paneles.

$\mathrm{GE}=$ Generación eléctrica $(\mathrm{Kw})$.

Potinver: Potencia del inversor (kWp).

$\mathrm{CTA}=$ Costo del $\mathrm{Kw}$ en tarifa actual.

$\mathrm{CTDAC}=$ Costo del Kw en tarifa DAC.

CT01 $=$ Costo del Kw en tarifa 01. actual \$.

EAACT=Estimado ahorro con tarifa EADAC $=$ Estimado ahorro con tarifa

\section{DAC \$}

EA01=Estimado ahorro con tarifa $01 \$$.

$\mathrm{AhN}=$ Ahorro neto $\$$.

$\mathrm{CON}=$ Consumo Kw/año.

ESFVI=Energía por sistema fotovoltaico interconectado Kw/año.

GESFVI= Generación de energia con sistema fotovoltaico.

fotovoltaico.

EFSFVI=Eficiencia del sistema

Estimada generación de kw/h

$$
D m * H S P * \text { Wpanel } * N p * 0.80 / 1000=G E
$$

Selección del inversor:

Np $*$ Wanel $/ 1000=$ Potinver

Proyectado de ahorro con tarifa actual:

$G E * C T A=E A A C T$

Proyectado de ahorro con tarifa DAC:

$G E^{*} C T D A C=E A D A C$

Proyectado de ahorro con tarifa 01:

$$
G E^{*} C T 01=E A 01
$$

Ahorro Neto:

$E A D A C-E A 01=A h N$

Eficiencia del sistema fotovoltaico \%:

$G E S F V I / C O N=E F S F V I$

\section{Resultados}

En la Tabla 3 se muestra la descripción del sistema a instalar cubriendo la energia brindada por CFE y la que se generará una vez puesto en marcha. 


\begin{tabular}{|l|r|l|}
\hline \multicolumn{3}{|c|}{ Descripción del sistema SFVI } \\
\hline Energía por CFE por & $14,596.00$ & Kw/año \\
\hline $\begin{array}{l}\text { Energía generada pra } \\
\text { SFVI }\end{array}$ & $15,186.40$ & Kw/año \\
\hline Potencia del sfvi & 9.63 & Kwp \\
\hline Eficiencia & 104.04 & $\%$ \\
\hline Potencia del inversor & 10 & Kwp \\
\hline $\begin{array}{l}\text { Capacidad de trabajo del } \\
\text { inversor }\end{array}$ & 96.25 & $\%$ \\
\hline Potencia del panel & 385 & Watts \\
\hline Número de paneles & 25 & Pz \\
\hline
\end{tabular}

Tabla 3 Descripción del SFVI

Fuente: Elaboración propia

En la tabla 4 se muestran los valores que se han realizado en la tarifa actual en este caso es DAC y las facturaciones actuales, así mismo se presentan las sumatorias de cada clausula donde también nos indica que el SFVI no podrá cubrir los consumos requeridos; pasa la misma situación en la parte facturable pero no todo está perdido ya que esto sucede en un determinado tiempo de 6 meses aproximadamente es decir lo que se acopla al sistema seguirá habiendo ciertas perdidas.

\begin{tabular}{|c|c|}
\hline Descripción & Total \\
\hline Consumo Kw/año & $14,596 \mathrm{kw} / \mathrm{año}$ \\
\hline Generación con sfvi kw/año & $15,186 \mathrm{kw} / \mathrm{año}$ \\
\hline Cargos fijos & $\$ 1,295.9$ \\
\hline DAP & $\$-$ \\
\hline Facturación sin IVA & $\$ 63,224.6$ \\
\hline Facturación actual & $\$ 74,843.9$ \\
\hline
\end{tabular}

Tabla 4 Los datos son de tarifa DAC

Fuente: Elaboración propia

En la tabla 5 se muestran las facturaciones actuales $y$ las proyecciones futuras que se pagaran en un determinado tiempo como a su vez también se observan los números en recuadro de color rojo donde se representan las pérdidas económicas que se están generando de manera continua por un lapso de tiempo de cerca de 6 meses, esta cantidad es permitible y conforme a la adaptación del sistema SFVI lo que coloquialmente se le menciona al cliente que en un año dejara de pagar a la red suministradora de energía eléctrica si mantiene sus consumos actuales.

\begin{tabular}{|l|r|}
\hline \multicolumn{3}{|c|}{ Descripción } & \multicolumn{1}{c|}{ Total } \\
\hline $\begin{array}{l}\text { Facturación bimestral con iva con } \\
\text { tarifa actual }\end{array}$ & $\$ 74,843.94$ \\
\hline $\begin{array}{l}\text { Facturación bimestral con sfvi con } \\
\text { tarifa acual }\end{array}$ & $\$ 77,306.01$ \\
\hline $\begin{array}{l}\text { Estimado ahorro con sfvi con tarifa } \\
\text { actual }\end{array}$ & $\$ 2,462.06$ \\
\hline $\begin{array}{l}\text { Facturación con cfe proyección dac } \\
100 \%\end{array}$ & $\$ 74,843.94$ \\
\hline $\begin{array}{l}\text { Facturación bimestral del sistema solar } \\
\text { proyección dac }\end{array}$ & $\$ 77,306.01$ \\
\hline $\begin{array}{l}\text { Estimado ahorro con sfvi proyección } \\
\text { dac }\end{array}$ & $\$ 2,462.06$ \\
\hline $\begin{array}{l}\text { Facturacion bimestral con proyección } \\
\text { tarifa 01 }\end{array}$ & $\$ 44,432.64$ \\
\hline
\end{tabular}

Tabla 5 Se muestran las facturaciones

Fuente: Elaboración propia

\section{Ejemplo 1}

En la tabla 6 se muestra la energía generada y suministrada por CFE, como se observan están en recuadros rojos debido que son perdidas, aunque son los meses con un alto índice de radiación no se alcanza a cubrir las necesidades, por lo consiguiente se obtienen perdidas económicas, pero no a gran escala por lo que se reduce hasta el 80 a $90 \%$ es decir que solo pagaríamos del 10 al 20\% de la factura actual.

Como se ha mostrado en las tablas 4 y 5 donde se indican que ciertas perdidas solo son por un periodo determinado, calculado para 6 meses después de esos 6 meses donde esas pérdidas se convierten en utilidades hasta la vida útil del SFVI que son de 25 a 30 años conforme a los cambios climáticos y mantenimiento que se brinda al menos una vez al año como mínimo para mantener la energía eléctrica funcional.

Esto se reduce si somos usuarios DAC donde se consume 3,225 $\mathrm{kWh}$ durante un bimestre se estará facturando por $\$ 17,318.72$ MXN pero que sucede si se tiene un SFVI donde no se generaran los $3,225 \mathrm{kWh}$ solamente $2,773.23 \mathrm{kWh}$ se tendrá $451.77 \mathrm{kWh}$ debajo del consumo, económicamente también es afectado, pero no a gran escala por lo cual los 2,773.23 kWh equivalen económicamente $\$ 14,927.48 \mathrm{MXN}$, como la factura es de $\$ 17,318.72 \mathrm{MXN}$ solo se paga las diferencia del consumo actual menos lo que se genera que son los $451.77 \mathrm{kWh}$ que son equivalentes a $\$ 2391.24 \mathrm{MXN}$, es lo que pagara en un futuro si contamos con SFVI aunque no se alcance la eficiencia energética. 
Pero se reduce de manera muy drástica la facturación; en este caso es del $86.20 \%$ por lo cual solo se pagó el $13.80 \%$ de la energía consumida en este caso, muchas personas preguntan ¿ qué sucede si tu sistema no cubre la eficiencia energética en los meses de poca radiación solar? es por ese motivo que se desarrolló este interfaz donde se muestran las peores condiciones que se ponen a prueba, en este sistema no hay mejor manera de explicarlo que de manera gráfica y numérica, para los clientes lo que les importa es pagar menos o simplemente no pagar un servicio tan esencial de manera monótona e inestable por lo que prefieren hacer un solo pago económico y la solución es unirse a este sistema de generación de energía fotovoltaica donde tu inversión si vale e importa; este ejemplo 1 es una ventana hacia dónde va direccionada la viabilidad ya sea energética o económica de nuestro sistemas fotovoltaicos.

\begin{tabular}{|l|r|}
\hline \multicolumn{1}{|c|}{ Descripción } & 13may19/10jul19 \\
\hline Consumo kw/h & $3225 \mathrm{kw} / \mathrm{h}$ \\
\hline Generación con SFVI kw/h & $2,773.23 \mathrm{kw} / \mathrm{h}$ \\
\hline Cargos fijos & -- \\
\hline DAP & $\$ 14,715.67$ \\
\hline Facturación sin IVA con CFE & $\$ 17,318.72$ \\
\hline Facturación con IVA con tarifa actual & $\$ 14,927.48$ \\
\hline $\begin{array}{l}\text { Facturaciòn bimestral con SFVI tarifa } \\
\text { actual }\end{array}$ & $-\$ 2,391.24$ \\
\hline Estimadoahorro con SFVI actual \$ con CFE & $\$ 17,318.72$ \\
\hline $\begin{array}{l}\text { Facturación con IVA } \\
\text { proyección DAC 100\% }\end{array}$ & $\$ 14,927.48$ \\
\hline $\begin{array}{l}\text { Facturaciòn bimestral delsistema solar } \\
\text { proyección DAC }\end{array}$ & $-\$ 2,391.24$ \\
\hline $\begin{array}{l}\text { Estimado ahorro con SFVI proyección } \\
\text { DAC }\end{array}$ & $\$ 10,024.89$ \\
\hline $\begin{array}{l}\text { Facturación bimestral con proyección } \\
\text { de tarifa 01 }\end{array}$ & \\
\hline
\end{tabular}

Tabla 6 Energía generada vs CFE

Fuente: Elaboración propia

\section{De las peores a mejores condiciones}

Como se vio en el ejemplo 1, donde se explican las eficiencias energéticas y económicas de los sistemas fotovoltaicos, aún hay más beneficios para los usuarios DAC o simplemente los que quieran adquirir este tipo de servicio, por lo que se añade un apartado donde se resuelve una pregunta inquietante para los usuarios ¿Qué pasara cuando deje de ser usuario DAC? y ¿qué beneficios obtendré a largo plazo?.
Cuando un usuario deje de ser usuario DAC entonces se convertirá en usuario básico es decir que estará en tarifa 01 ya que reducirá su consumo de manera drásticas, si nos basamos en la tabla 6 se muestra que se realizó un consumo de 3,225 kWh por lo cual se está pagando $\$ 17,318.72$ MXN una vez que entremos en tarifa 01 , solo se realiza la factura de \$10,024.89 MXN aun así teniendo el mismo consumo. Recordemos que el sistema fotovoltaico o SFVI genera una potencia menor que $3,225 \mathrm{kWh}$, que es de $2,773.23 \mathrm{kWh}$, pero su equivalencia económica es de $\$ 14,927.48$ MXN estando en tarifa DAC, la diferencia que existe entre 3,225 kWh y 2,773.23 kWh es de $451.77 \mathrm{kWh}$ que su equivalencia en tarifa DAC es de \$2391.24 MXN como se nota desde los $\$ 17,318.72 \mathrm{MXN}$ que se paga, se facturara a $\$ 2,391.24 \mathrm{MXN}$ aun con tarifa DAC pero una vez pasando a tarifa 01 esos $\$ 2,391.24$ MXN se convertirá a $\$ 856.60 \mathrm{MXN}$ y serán fijos como se muestra en la Tabla 7; esto quiere decir que tenemos una eficiencia económica del $95.06 \%$ y una eficiencia energética del $86 \%$ es decir que solo pagaremos el $4.94 \%$ de la energía consumida en el bimestre del 13 mayo al 10 julio, y su equivalencia es de $\$ 856.60 \mathrm{MXN}$.

Estos beneficios se obtienen cuando la generación de energía eléctrica es menor que el consumo entonces ¿que pasara cuando el sistema fotovoltaico supere el consumo o la energía que brinda CFE?, como se muestra en la Tabla 8 no tenemos números rojos; es decir que en ese bimestre no habrá perdidas sino todo lo contrario tenemos una eficiencia energética del 119\%, eficiencia económica con tarifa DAC del $119 \%$ es decir que se tendrá un saldo a favor de \$2,160.63MXN esto es equivalente del $19 \%$ de utilidad. Lamentablemente esto sucederá solo una vez por lo que CFE pagara los excedentes que se generan con el SFVI conforme a la tarifa en la que nos encontremos; es decir que estos $\$ 2,160.63 \mathrm{MXN}$ se convertirán en \$881.99 MXN cuando estemos en tarifa 01, lo cual nos cambiaran en energía por lo que su equivalencia es $459.77 \mathrm{kWh}$ como se muestra en la tabla 9.

\begin{tabular}{|r|r|}
\hline \multicolumn{2}{|c|}{ Tarifa 01 } \\
\hline Consuno kwh & Facturación \\
\hline $451.77 \mathrm{kwh}$ & $\$ 856.60$ \\
\hline
\end{tabular}

Tabla 7 Facturación en tarifa 01

Fuente: Elaboración propia 


\begin{tabular}{|l|r|}
\hline \multicolumn{1}{|c|}{ Descripción } & 9marz20/8may20 \\
\hline Consumo kw/h & 2455 \\
\hline Generación con SFVI kw/h & $2,914.45$ \\
\hline Cargos fijos & $\$ 216.10$ \\
\hline DAP & $\$ 9,952.57$ \\
\hline Facturación sin IVA con CFE & $\$ 11,795.66$ \\
\hline Facturación con IVA con tarifa actual & $\$ 13,956.29$ \\
\hline $\begin{array}{l}\text { Facturaciòn bimestral con SFVI tarifa } \\
\text { actual }\end{array}$ & $\$ 2,160.63$ \\
\hline Estimadoahorro con SFVI actual\$ & $\$ 11,795.66$ \\
\hline $\begin{array}{l}\text { Facturación con iva con cfe proyección } \\
\text { dac 100\% }\end{array}$ & $\$ 13,956.29$ \\
\hline $\begin{array}{l}\text { Facturaciòn bimestral delsistema solar } \\
\text { proyección DAC }\end{array}$ & $\$ 2,160.63$ \\
\hline $\begin{array}{l}\text { Estimado ahorro con SFVI proyección } \\
\text { DAC }\end{array}$ & $\$ 7,479.27$ \\
\hline $\begin{array}{l}\text { Facturación bimestral con proyección de } \\
\text { tarifa 01 }\end{array}$ & \\
\hline
\end{tabular}

Tabla 8 Generacion del SFVI mayor que la que brinda CFE

Fuente: Elaboración propia

\begin{tabular}{|r|r|}
\hline \multicolumn{2}{|c|}{ Tarifa 01 } \\
\hline Consuno kwh & Facturación \\
\hline $459.45 \mathrm{kwh}$ & $\$ 881.99$ \\
\hline
\end{tabular}

Tabla 9 Facturación en tarifa 01

Fuente: Elaboración propia

En el Gráfico 1, se presenta una comparación de manera anual de la energía que proporciona CFE y de la que se genera con el SFVI descrito en la Tabla 3.

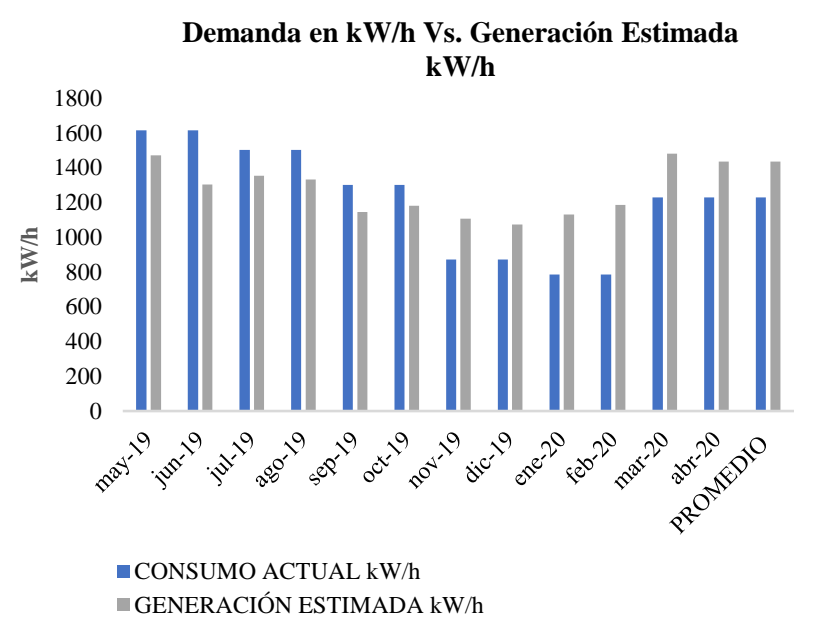

Grafíco 1 Generación de energía de CFE vs SFVI Fuente: Elaboración propia.

El sistema contiene una hoja independiente de la radiación solar de cada estado de la República Mexicana, como se muestra en la tabla 10, esto es muy importante para realizar un dimensionamiento fotovoltaico.

\begin{tabular}{|l|c|r|c|c|c|c|}
\hline \multicolumn{1}{|c|}{ Estado } & \multicolumn{1}{c}{ En } & \multicolumn{1}{c}{ Feb } & \multicolumn{1}{c|}{ Mar } & \multicolumn{1}{c|}{ Abr } & May & Jun \\
\hline Puebla & 4.73 & 5.5 & 6.2 & 6.21 & 6.16 & 5.64 \\
\hline Jul & Ago & Sept & Oct & Nov & Dic & \\
\hline \multicolumn{2}{|c|}{5.67} & 5.57 & 4.95 & 4.94 & 4.79 & \multicolumn{4}{c|}{4.49} \\
\hline
\end{tabular}

Tabla 10 Radiación de cada estado de Puebla Fuente: nasa.com

\section{Punto de equlibrio}

Esta parte es muy fundamental para cada proyecto ya que el cliente determinara invertir o no en un proyecto, ya que muestra el tiempo de recuperación de la inversión hablando económicamente; es decir que si el proyecto costo \$220,007.34 MXN, pero el sistema fotovoltaico genera 77,306.01MXN anuales, por lo tanto, el tiempo de recuperación es de 2.8 años se obtiene dividiendo costo del proyecto entre la generación del proyecto de manera anual económicamente hablando, como se observa en la tabla 11, así mismo se muestra en la gráfica 2 el tiempo de retorno de la inversión del sistema en inversión DAC.

\begin{tabular}{|c|r|l|}
\hline Costo del proyecto & $\mathbf{\$ 2 2 0 , 0 0 7 . 3 4}$ & \multicolumn{1}{c|}{ MXN } \\
\hline Generación del proyecto & $\$ 77,306.01$ & MXN \\
\hline Tiempo de recuperación & 2.8 & AÑOS \\
\hline
\end{tabular}

Tabla 11 Punto de equilibrio del proyecto Fuente: Elaboración propia

Recuperación de inversión con tarifa DAC

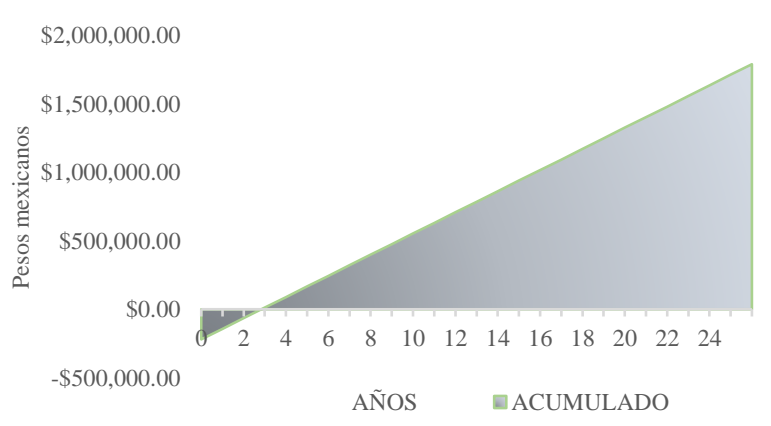

Gráfico 2 Tiempo de retorno de inversión Fuente: Elaboración propia

\section{Agradecimiento}

A la Universidad Politécnica de Amozoc por el "Apoyo a la Investigación Científica y Desarrollo Tecnológico" y por gestionar los recursos ante las instancias correspondientes para el financiamiento del proyecto. 


\section{Conclusiones}

En este proyecto se desarrolló una calculadora con el programa Excel con el objetivo de dar a conocer el funcionamiento de los cálculos fotovoltaico y para obtener los dimensionamientos de sistemas fotovoltaicos interconectados a la red de CFE, especialmente para pasar de la tarifa DAC a la tarifa 01, al lograr este cambio de tarifas se reduce el pago económico a CFE, en el ejemplo que se trabajo con el consumo de 3,225 kWh por lo cual se estaba pagando $\$ 17,318.72$ al realizar los cálculos con la calculadora se redujo el consumo a $451.77 \mathrm{kWh}$ pagando solo $\$ 856.60$ ya en la tarifa 01, esto quiere decir que tenemos una eficiencia económica del $95.06 \%$ y una eficiencia energética del $86 \%$. También con la calculadora se obtiene automáticamente el costo del proyecto y el retorno de inversión, para el ejemplo utilizado el retorno es de 2.8 años. En la calculadora al cambiar la potencia del panel se obtiene automáticamente los nuevos cálculos. En el proyecto se observó que una de las partes importantes fue el área financiera donde se explicó la parte del punto de equilibrio de la forma más práctica y las utilidades que obtendremos en adquirir un SFVI.

\section{Recomendaciones}

- Respetar la normativa y reglamentos electrotécnicos aplicables a instalaciones eléctricas.

- Proteger de la intemperie los equipos electrónicos en cajas que cumplan con la protección IP65. El cableado de la instalación debe cumplir con la normativa eléctrica vigente, minimizando las caídas de tensión.

- Aumentar la biblioteca de la calculadora con las características eléctricas de los paneles solares y de los inversores.

\section{Referencias}

Almanza-Fundora, I., García-Fernández, R. R., \& García-Reina, F. (2019). Desarrollo de un sistema para la medición de la eficiencia energética de celdas solares. Revista Ciencias Técnicas Agropecuarias, 28(1).
Propergol, S. (2019). Manual avanzado EXCEL 2019. Anaya Multimedia.

Canarias, G. D. (2018). Instalaciones fotovoltaicas. Obtenido de http://www.agenergia.org/files/resourcesmodul e/@random49914e4ed9045/1234263307_Guia FotovoltaicaGobCan.pdf.

Fletes, N., Paredes, C., Dibene, L. (2016). Eficiencia en la producción de energía de un panel fotovoltaico a diferente inclinación en Nuevo Vallarta, Nayarit. Revista de Aplicaciones de Ingeniería, 3.

Peña, R. (2015). Excel 2016: Manual práctico paso a paso. ALTARIA

Flores, O., González, R., Juárez, V., Huitzil, I. (2016). Análisis comparativo de rendimiento, costo y producción energética entre el sistema aislado e interconectado de la empresa cartón corrugado Puebla S.A. de C.V. Revista de Aplicaciones de Ingeniería, 3(9).

Flores, O., Huitzil, I., González, R. y Hernández, A. (2017). Reducción de la eficiencia energética del parque solar UPAM por suciedad y deposición de polvo. Revista de Innovación Sistemática, 1(3), 26-36.

Burrueco, D. (2016). Tablas dinámicas con Excel 2016. RA-MA.

Flores, O., Huesca, E., Pacheco, E.A. y Aguilar, J.M. (2018). Dimensionamiento, instalación y puesta en marcha del Parque Solar UPAM de $50 \mathrm{~kW}$ de potencia. Revista de Energías Renovables, 2(5), 1-13

Mertens, K. (2014). Photovoltaics Fundamentals, Technology and Practice). United Kingdom: John Wil ey \& Sons Ltd.

Fletes, N., Paredes, C., Dibene, L. (2016). Eficiencia en la producción de energía de un panel fotovoltaico a diferente inclinación en Nuevo Vallarta, Nayarit. Revista de Aplicaciones de Ingeniería, 3.

Harper, E. (2012). Instalaciones y sistemas fotovoltaicos. Limusa. 
García, M., Marroyo, L., Lorenzo, E., Pérez, M. (2011). Soiling and other optical losses in solartracking PV plants in Navarra. Progr. Photovolt: Res. Appl. 19 (2), pp. 211-217.

Martínez J. (2011). Implementación de un Sistema de Celdas Fotovoltaicas para el Alumbrado de Automatización de la Facultad de Ingeniería de UAQ. Recuperado de http://ri.uaq.mx/bitstream/123456789/1227/1/R I000800.pdf

Daniel Almarza, A. G. (marzo de 2017). Guía de buenas y malas prácticas de instalaciones fotovoltaicas sobre techos. Documento anexo a la. Obtenido de http://www.sec.cl/pls/portal/docs/PAGE/SEC20 05/ELECTRICIDAD_SEC/ERNC/GENERACI ON_DISTRIBUIDA/LINKS_Y_NOTICIAS/T AB6243717/GU\%CDA-DE-BUENAS-YMALAS-PR\%C1CTICAS-DEINSTALACIONES-FOTOVOLTAICAS..PDF

Liqun, L., Zhiqi, L., Chunxia, S.Z.L. (2012). Degraded output characteristic at atmospheric air pollution and economy analysis of PV power system: a case study. Przegl. Elektrotech. (Electr. Rev.) 88 (9A), pp. 281-284.

Enríquez, G. (2010). El ABC de las Instalaciones Eléctricas en Sistemas Eólicos y Fotovoltaicos. Limusa.

Anguiano J. (2012). Constante solar. P.15 - 17 Impacto de la temperatura de los módulos en la eficiencia de un sistema fotovoltaico conectado a la red. Recuperado de: http://148.206.53.84/tesiuami/UAMI16030.pdf

Llerena-Pizarro, O., Proenza-Perez, N., Tuna, C. E., \& Silveira, J. L. (2020). A PSO-BPSO Technique for Hybrid Power Generation System Sizing. IEEE Latin America Transactions, 18(08), 1362-1370.

De la Cruz Fernández, E. (2020). Diseño de un sistema eléctrico aislado utilizando el software HOMER para electrificar el caserío Santa Rosa de Tumar en el distrito de Huambos, provincia de Chota-Cajamarca.

Lindao Suárez, W. E. (2020). Propuesta de diseño de un sistema de energía solar fotovoltaica. Caso de aplicación en casa comunal de Cooperativa Los Paracaidistas en la ciudad de Guayaquil.
Lizana Rivera, C. R. (2020). Dimensionamiento de un sistema con recursos energéticos renovables conectado a red en el Distrito de Tarapoto, San Martin.

Cabrejos Piscoya, P. A. (2020). Dimensionamiento de un sistema fotovoltaico domiciliario para el caserío El Limonar, distrito de Jayanca, provincia de Lambayeque, departamento de Lambayeque. 